\title{
PETROPHYSICAL PARAMETERS ESTIMATION USING LEVENBERG- MARQUARDT AND SINGULAR VALUE DECOMPOSITION INVERSION SCHEMES
}

\author{
Moataz Mohamed Gomaa Abdelrahman \\ PhD student, University of Miskolc, Department of Geophysics \\ Ain shams University, Department of Geophysics \\ 3515 Miskolc, Miskolc-Egyetemváros, e-mail: $\underline{\text { mh.geophysicist@sci.asu.edu.eg }}$ \\ Norbert Péter Szabó \\ professor, University of Miskolc, Department of Geophysics \\ MTA-ME Geoengineering Research Group \\ 3515 Miskolc, Miskolc-Egyetemváros, e-mail: norbert.szabo.phd@gmail.com
}

\section{Mihály Dobróka}

professor emeritus, University of Miskolc, Department of Geophysics

3515 Miskolc, Miskolc-Egyetemváros, e-mail:dobroka@uni-miskolc.hu

\begin{abstract}
Well logging inversion was carried out using Levenberg-Marquardt (LM) and Singular Value Decomposition (SVD) techniques for the determination of petrophysical parameters, respectively. In this research, synthetic data contaminated with $5 \%$ Gaussian noise, and field data were used to compare the results from the two inversion methods. MATLAB software has been developed to solve the overdetermined inverse problem. The estimated petrophysical parameters from both inversion methods had been compared to one another in terms of robustness to noise, rock interface differentiation, different fluid prediction, and the accuracy of the estimated parameters. This research returns the reason to the inner iterative loop which is considered more about the Jacobian matrix sensitivity. The inversion results showed that both methods can be used in petrophysical data estimation for a reliable well-log data interpretation.
\end{abstract}

Keywords: Forward modeling, Local inversion, MATLAB, Borehole geophysics

\section{Introduction}

Borehole geophysics is one of the most common methods in geophysical surveys, which can provide information about the different types of rocks that are encountered by the drilling process. The evaluation of the acquired data from open-hole logging enables to estimate some petrophysical parameters such as porosity, shale volume, and fluid saturation. Several industrial fields are counting on the data from the borehole geophysics to construct or estimate a geological model such as oil industry or groundwater exploration.

The inversion process can give a quick and accurate evaluation of the well-logging data, counter to the deterministic methods which need sequential steps, and each step depends on the previous result. In inverse modeling, the petrophysical parameters could be estimated automatically together at the same time with indicating the error percentage (Dobróka et al., 2016). The two common inversion methods are the local and the interval methods, the two methods that were used in this research are considered types of local inversion. In the local inversion method, the petrophysical parameters are estimated at the 
same depth using the data acquired from the different logging tools. In the forward modeling phase, an initial model is constructed using the response functions, which in turn will be optimized to decrease the difference between the actual and the theoretical data (Szabó and Dobróka, 2020). In this research, the initial model was refined using the LM and SVD schemes and the results were compared for three models (5\% Gaussian noise synthetic model, and real measured data). The most important aim of the inversion process is to extract the most reliable and accurate parameters. The noise of the data highly affects the quality of the estimated parameters; then, the degree of the accuracy of the evaluation of the borehole data is very important to give a better understanding of the rock properties. Therefore, in the case of the LM method, the relative data distance was estimated at each iteration, while in the SVD a threshold value has been chosen to stop the optimization process.

Understanding the well-logging inversion procedure requires, in the beginning, to realize the aim from the evaluation of well-logging data as well as the relationship between the model parameters and measurement data (Szabó and Dobróka, 2013). Consequently, the final aim of the inversion process is to get a geological interpretation of the rock interface, in other words, getting a separation between different formations, estimate the thickness of the layers, and calculate the different petrophysical parameters such as porosity, fluid content, and shale volume. According to (Dobróka and Szabó, 2011) and (Szabó, 2018) well-logging inversion can be considered as a joint inversion procedure with two groups of parameters. The first group of parameters so-called the zone parameters, do not have a significant change with depth interval (e.g., saturation exponent, cementation exponent, and water resistivity). The second group can be formed by the parameters called the layer parameters, which can be nearly fixed through a given layer but change from one to another (e.g., mineral composition, shale volume, and permeability).

\section{Theoretical Background}

In hydrocarbon exploration, a combination of logging tools called triple combo or quad combo is usually used. This combination consists of lithology, porosity, and fluid saturation sensitive tools. Through this section, the theoretical background of the forward modeling and local inversion based on two different optimization techniques (LM and SVD) are going to be discussed.

\subsection{Forward modeling}

The relationship between the logging data and the petrophysical properties can be expressed in the form so-called response function. The following equations represent the response function used for data calculation and initial forward model construction (GR, $\rho b, \emptyset_{N}, P e, R_{d}$, and $R_{s}$ are gamma-ray, density, neutron porosity, photo-electric, deep resistivity, and shallow resistivity logs respectively) (Szabó, 2018; Drahos, 2005; Szabó, 2011; Alberty and Hashmy, 1984):

$$
\begin{gathered}
G R=\rho_{b}{ }^{-1}\left(V_{s h} G R_{s h} \rho_{s h}+\sum_{i=1}^{N} V_{m a, i} G R_{m a, i} \rho_{m a, i}\right), \\
\rho_{b}=\emptyset\left[\rho_{m f}-1.07\left(1-S_{x o}\right)\left(\alpha_{0} \rho_{m f}-1.24 \rho_{h}\right)\right]+V_{s h} \rho_{s h}+\sum_{i=1}^{N} V_{m a, i} \rho_{m a, i},
\end{gathered}
$$




$$
\begin{aligned}
& \emptyset_{N}=\emptyset\left\{\begin{array}{c}
\emptyset_{N . m f}-\left(1-S_{x 0}\right) C_{c o r} \\
-2 \emptyset\left(1-S_{x 0}\right)-S_{h f}\left(1-2.2 \rho_{h}\right) \\
.\left[1-\left(1-S_{x 0}\right)\left(1-2.2 \rho_{h}\right)\right]
\end{array}\right\}+V_{s h} \rho_{s h}+\sum_{i=1}^{N} V_{m a, i} \emptyset_{m a, i} \\
& P_{e}=\frac{1.07}{\rho_{b}+0.19}\left[\varnothing S_{x 0} U_{m f}+\emptyset\left(1-S_{x 0}\right) U_{h}+V_{s h} U_{s h}+\sum_{i=1}^{N} V_{m a, i} U_{m a, i}\right] \\
& \frac{1}{\sqrt{R_{d}}}=\left[\frac{V_{s h}^{\left(1-0.5 V_{s h}\right)}}{\sqrt{R_{s h}}}+\frac{\sqrt{\emptyset}^{m}}{\sqrt{a R_{w}}}\right]{\sqrt{S_{w}}}^{n} \\
& \frac{1}{\sqrt{R_{s}}}=\left[\frac{V_{s h}{ }^{\left(1-0.5 V_{s h}\right)}}{\sqrt{R_{s h}}}+\frac{\sqrt{\varnothing}^{m}}{\sqrt{a R_{m f}}}\right]{\sqrt{S_{x 0}}}^{n} \text {, } \\
& \varnothing+V_{s h}+\sum_{i=1}^{N} V_{m a, i}=1,
\end{aligned}
$$

where $V_{m a}, i(\mathrm{v} / \mathrm{v})$ refer to the fractional volume of the $i$-th matrix constituent [9], The total number of mineral components is defined via $N$, the fractional volume of pore spaces that are free of shale is labeled by $\varnothing(\mathrm{v} / \mathrm{v})$, where $\emptyset_{N S}$ (neutron value in front of a thick volume of pure sandstone rock) $\neq 0$ because the neutron log is calibrated for limestone, not for sandstone, and the water saturation fraction in the invaded and uninvaded areas are $S_{x 0}$ and $S_{w}$ respectively. The physical properties of mud filtrate $(\mathrm{mf})$, hydrocarbon $(h)$, shale $(s h)$, mud filtrate correction coefficients $\left(\mathrm{C}_{\mathrm{cor}}\right)$, mud filtrate coefficient $\left(\alpha_{0}\right), \mathrm{m}$ is the cementation exponent $(m)$, saturation exponent $(n)$ and the rock matrix $(m a)$ are expressed by the zone parameters in equations 1 to 7 . As prementioned, these zone parameters are considered constants during the forward modeling phase. These zone parameters could be provided from special and conventional core analysis tests, drilling information, or graphics processing techniques. Table 1 shows the actual values of zone parameters. The Indonesian formulae (eq. 6 and 7) were used for calculating the resistivities of uninvaded and invaded zones $R_{d}$ and $R_{s}$, respectively. Equation 8 is the material balance equation which, represents a constraint in solving the inverse problem.

\subsection{Local Inversion}

Before the local inversion has been started, the data must be prepared in matrix form. The data preparation consists of grouping the petrophysical parameters for shaly-sand formations in column parameters vector:

$$
m=\left[\emptyset, V_{s h}, V_{V s d}, S_{w}, S_{x 0}\right]^{T},
$$

where $\mathrm{T}$ is the symbol of transpose. And by the same way the measured well-logging data at the same depth are represented in a column data vector:

$$
d^{(0)}=\left[G R, \emptyset_{N}, \rho_{b}, R_{S}, R_{d}, P_{e}\right]^{T}
$$


The length of the data vector is larger than the model parameters vector, which defines the inverse problem of borehole geophysics as an overdetermined problem (Dobróka and Szabó, 2011). The relationship between the calculated data and model parameters can be expressed as a nonlinear relationship as (Menke, 1984):

$$
d^{(c)}=g(m),
$$

where $\mathrm{g}$ is the response function, that is used for calculating the well-logging data at a certain depth.

Table 1. Zone parameters are used for the traditional processing of borehole geophysics (Szabó and Dobróka, 2020).

\begin{tabular}{|l|c|c|c|c|}
\hline Well log & Zone Parameter & Symbol & Value & $\begin{array}{c}\text { Dimensional } \\
\text { unit }\end{array}$ \\
\hline Natural gamma-ray & Sand & $\mathrm{GR}_{\mathrm{sd}}$ & 10 & $\mathrm{API}$ \\
\hline \multirow{4}{*}{ Gamma-gamma (density) } & Shale & $\mathrm{GR}_{\mathrm{sh}}$ & 154 & $\mathrm{API}$ \\
\cline { 2 - 5 } & Sand & $\rho_{\mathrm{sd}}$ & 2.65 & $\mathrm{~g} / \mathrm{cc}$ \\
\cline { 2 - 5 } & Shale & $\rho_{\mathrm{sh}}$ & 2.54 & $\mathrm{~g} / \mathrm{cc}$ \\
\cline { 2 - 5 } & Mud filtrate & $\rho_{\mathrm{mf}}$ & 1.02 & $\mathrm{~g} / \mathrm{cc}$ \\
\hline \multirow{5}{*}{ Neutron porosity } & Hydrocarbon(gas) & $\rho_{\mathrm{h}}$ & 0.2 & $\mathrm{~g} / \mathrm{cc}$ \\
\cline { 2 - 5 } & Sand & $\Phi_{\mathrm{N}, \mathrm{sd}}$ & -0.04 & $\mathrm{v} / \mathrm{v}$ \\
\cline { 2 - 5 } & Shale & $\Phi_{\mathrm{N}, \mathrm{sh}}$ & 0.31 & $\mathrm{v} / \mathrm{v}$ \\
\cline { 2 - 5 } & Mud filtrate & $\Phi_{\mathrm{N}, \mathrm{mf}}$ & 0.95 & $\mathrm{v} / \mathrm{v}$ \\
\cline { 2 - 5 } & mf correction coefficients & $\mathrm{C}_{\mathrm{cor}}$ & 0.69 & - \\
\cline { 2 - 5 } & Residual hydrocarbon & $\mathrm{S}_{\mathrm{hrf}}$ & 1.2 & - \\
\cline { 2 - 5 } & Shale & $\mathrm{R}_{\mathrm{sh}}$ & 1 & $\mathrm{Ohm} . \mathrm{m}$ \\
\cline { 2 - 5 } & Pore water & $\mathrm{R}_{\mathrm{w}}$ & 0.5 & $\mathrm{Ohm} . \mathrm{m}$ \\
\cline { 2 - 5 } & Mud filtrate & $\mathrm{R}_{\mathrm{mf}}$ & 0.28 & $\mathrm{Ohm} \cdot \mathrm{m}$ \\
\cline { 2 - 5 } & Cementation exponent & $\mathrm{m}$ & 1.5 & - \\
\cline { 2 - 5 } & Saturation exponent & $\mathrm{n}$ & 1.8 & - \\
\cline { 2 - 5 } & Tortuosity factor & $\mathrm{a}$ & 1 & - \\
\hline Photo-electric & Sand & $\mathrm{U}_{\mathrm{sd}}$ & 4.8 & $\mathrm{Barn} / \mathrm{cm}^{3}$ \\
\cline { 2 - 5 } & Shale & $\mathrm{U}_{\mathrm{sh}}$ & 9 & $\mathrm{Barn} / \mathrm{cm}^{3}$ \\
\cline { 2 - 5 } & Mud filtrate & $\mathrm{U}_{\mathrm{mf}}$ & 0 & $\mathrm{Barn} / \mathrm{cm}^{3}$ \\
\cline { 2 - 5 } & Hydrocarbon(gas) & $\mathrm{U}_{\mathrm{h}}$ & 0 & $\mathrm{Barn} / \mathrm{cm}^{3}$ \\
\hline
\end{tabular}

The objective function to be optimized is (Szabó, 2012):

$$
D=\sqrt{\left[\frac{1}{N} \sum_{k=1}^{N}\left(\frac{d_{k}^{(o b s)}-d_{k}^{(c a l)}}{d_{k}^{(o b s)}}\right)^{2}\right]} * 100(\%) .
$$

where $d_{k}^{(o)}$ and $d_{k}^{(c)}$ are the measured or observed and calculated data, respectively, where $N$ is the number of inverted data.

\subsubsection{Levenberg-Marquardt (LM)}

LM method can be considered as a linear inversion method, where the initial model is iteratively refined until the best fitting is achieved (Marquardt, 1963). The model update equation can be expressed as: 


$$
\left(G\left(m^{i}\right)^{T} G\left(m^{i}\right)+\lambda I\right) \delta m=-G\left(m^{i}\right)^{T} \delta d,
$$

where $\lambda$ is a damping factor by training it could range from 100 to 0.01 . The function of this damping factor is to overcome the singular matrix problem of the term $G\left(m^{i}\right)^{T} G\left(m^{i}\right) . I$ is the identity matrix, $G(m)$ is the sensitivity matrix or Jacobian matrix, $\delta m$ is ( $M$ (number of parameters) $x_{I}$ ) parameters updates vectors, and $\delta d$ is $\left(N_{l}\right.$ (number of well $\left.\left.\operatorname{logs}\right) x_{l}\right)$ the difference between calculated and observed data. Meanwhile, the $G(m)$ (Jacobian matrix) could be calculated numerically by the finite difference method to obtain the partial derivatives of parameters with each response function $(f(m))$, the $G(m)$ can be written as:

$$
G(m)=\left[\begin{array}{ccc}
\frac{\delta f_{1}(m)}{\delta\left(m_{1}\right)} & \ldots & \frac{\delta f_{1}(m)}{\delta\left(m_{M}\right)} \\
\vdots & \ddots & \vdots \\
\frac{\delta f_{N}(m)}{\delta\left(m_{1}\right)} & \cdots & \frac{\delta f_{N l}(m)}{\delta\left(m_{M}\right)}
\end{array}\right]
$$

the iterative scheme of LM can be concluded in the following equation to update the model parameter (Heriyanto and Srigutomo, 2017):

$$
m^{i+1}=m^{i}+\left(G\left(m^{i}\right)^{T} G\left(m^{i}\right)+\lambda I\right)^{-1} G\left(m^{i}\right)^{T} \delta d,
$$

\subsubsection{Singular Value Decomposition Scheme}

(Meju, 1992) has formulated an iterative equation of model parameter update using the idea of factorizing the Jacobian matrix into three other matrices as follow:

$$
G\left(m^{i}\right)=U S V^{T},
$$

where $U$ is $(N x M)$ data eigenvector, $V$ is $(M x M)$ model parameters eigenvector, and $S$ is $(M x M)$ matrix with eigenvalues in its diagonal. These positive values represent the eigenvalues of $G(m)$ matrix with i $\leq \mathrm{M}$. The SVD based inversion method standing on the idea stated that the matrix produced from the term $G\left(m^{i}\right)^{T} G\left(m^{i}\right)$ is said to be ill-conditioned in case of small eigenvalues. Therefore, the SVD scheme suggested adding a positive bias to the eigenvalues of the term $G\left(m^{i}\right)^{T} G\left(m^{i}\right)$. By substitute equation (16) into equation (13):

$$
\left(V S^{2} V^{T}+\lambda I\right) \delta m=-\left(V S U^{T}\right) \delta d,
$$

by applying the idea of adding the damped factor to the diagonal non zero values [15], the left-hand side of equation (17) can be written as:

$$
\left(V S^{2} V^{T}+\lambda I\right)=\left(V \operatorname{diag}\left(\eta_{i}{ }^{2}\right) V^{T}\right)+\lambda^{2} I=V \operatorname{diag}\left(\eta_{i}{ }^{2}+\lambda^{2}\right) V^{T},
$$

where $\eta i$ is the $i$-th eigenvalue, the following equation shows the inverse of equation (18):

$$
\left(V \operatorname{diag}\left(\eta_{i}^{2}+\lambda^{2}\right) V^{T}\right)^{-1}=V \operatorname{diag}\left\{\frac{1}{\eta_{i}^{2}+\lambda_{i}^{2}}\right\} V^{T} .
$$

Consequently, the final equation to update the model parameters provided by (Ekinci and Demirci, 2008) can be written as: 


$$
m^{i+1}=m^{i}+V \operatorname{diag}\left\{\frac{\eta_{i}}{\eta_{i}^{2}+\lambda_{i}^{2}}\right\} U^{T} \delta d .
$$

The damping factor has been developed by comparing the misfit of the $L$-th test at any iteration and $(L+1)$-th test; if the misfit has been enhanced, it will be accepted. This method called the RIDGE method, where a biased positive value has been added to the diagonal matrix eigenvalues (Arneson and Hersir, 1988), according to the following equation:

$$
\lambda=\eta_{L} \Delta X^{\frac{1}{L}}
$$

where $\Delta X$ can be given by:

$$
\Delta X=\frac{X_{R-1}-X_{R}}{X_{R-1}}
$$

where $X_{R-l}$ and $X_{R}$ are the previous and present iterations misfit.

\section{Results}

In this research, 5\% Gaussian noise synthetic data, and field data were used to apply both LM and SVD based inversion. The misfit between the calculated and measured data was calculated using equation 12 of relative data distance as prementioned in section 2.2.

\subsection{Synthetic data}

The LM and SVD were applied on the 4-layers model (shaly sand, sand, shale, and sand), where the synthetic model supposed the presence of hydrocarbon and water zones. There are sublayers inside layer 1 to represent the transform inside the layer from sand to shale. The data vector that resulted from the forward model belongs to GR, $\rho b, \emptyset_{N}$, Rs, and Rd. Figure (1) shows the forward well-logging data derived from equations 1 to 8 of $25 \mathrm{~m}$ of rocks, the right side shows the diagrammatic block of the 4layer earth model and the fluid distribution. The predefined model shows that layer 1 consists of sandshale sequence with hydrocarbon filling the pore spaces, while layer 2 shows one sand layer filled with hydrocarbon then water, finally layers 3 and 4 show no change in fluid type (water) but the lithology change from shale to sand; so, the number of minors layers is 7 . The synthetic model suggested the presence of hydrocarbon and water where the Oil-Water Contact (OWC) or the Gas-Water Contact (GWC) lies at depth of $9 \mathrm{~m}$.

The data calculated from the pre-mentioned response functions were inverted using LM and SVD schemes. The results obtained show a small relative data distance, which can be seen in table 2. Inversion results depend on the initial model. If the initial model was too far from the solution, the inversion problem may be trapped in a local minimum and produce a high relative data distance. In the overdetermined inversion problem, there is another problem that may cause deviation from the real model parameters; this problem is the response function itself which produces overestimated or underestimated model parameters to decrease the misfit between real and calculated data. Therefore, the SVD method used the RIDGE criteria to avoid this problem. 


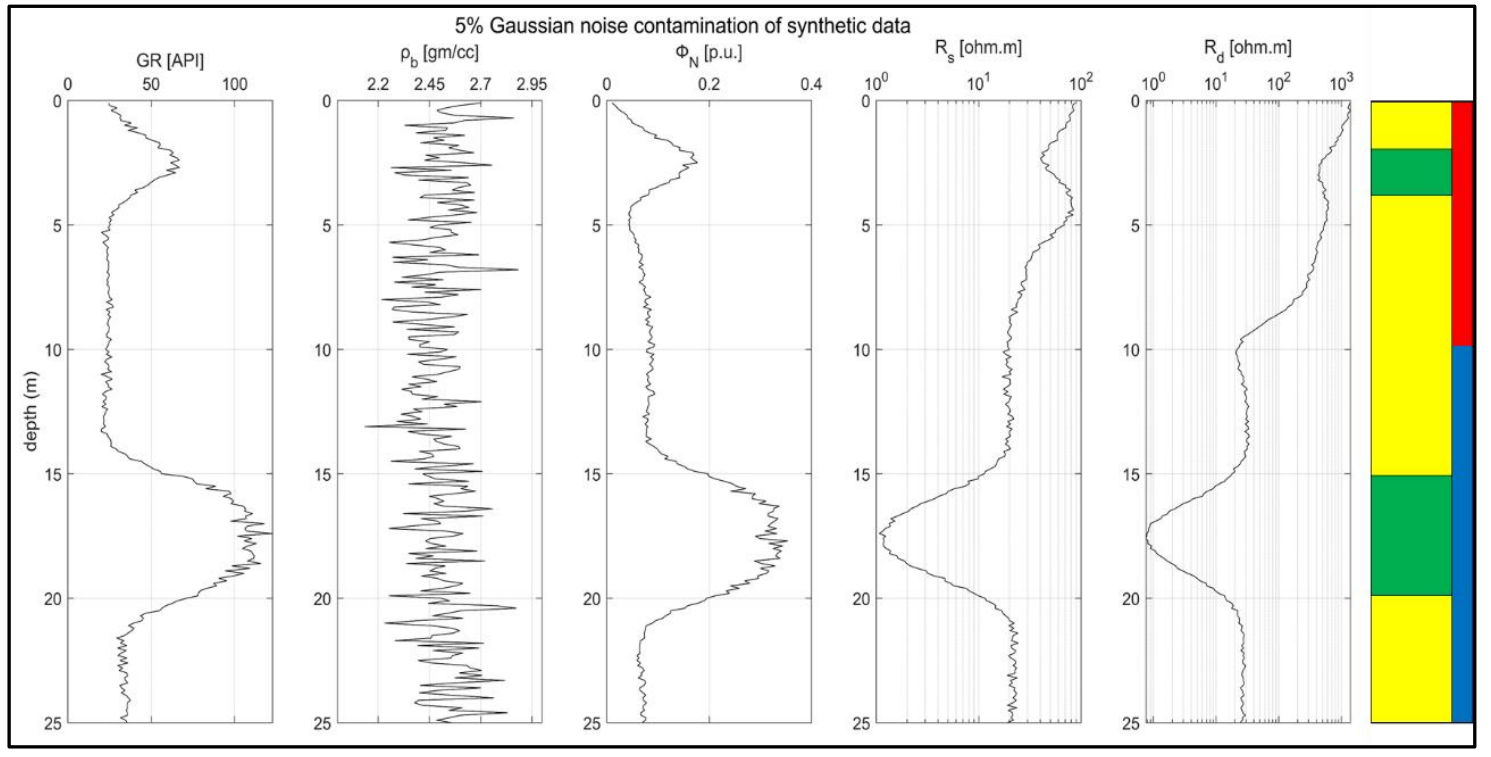

Figure 1. Synthetic well-logging data with $5 \%$ Gaussian noise, the right schematic block shows the predefined layers model (Sand (yellow), shale (green), and fluid distribution (hydrocarbon (red), water (blue)).

Table 2. True and estimated model parameters using LM and SVD.

\begin{tabular}{c|ccc|ccc|cccc}
\hline \multicolumn{3}{c}{ True } & \multicolumn{3}{c}{ LM } & \multicolumn{3}{c}{ SVD } \\
\hline Layers & $\Phi$ & $\mathrm{V}_{\text {sh }}$ & $\mathrm{S}_{\mathrm{w}}$ & $\Phi$ & $\mathrm{V}_{\text {sh }}$ & $\mathrm{S}_{\mathrm{w}}$ & $\Phi$ & $\mathrm{V}_{\text {sh }}$ & $\mathrm{S}_{\mathrm{w}}$ \\
\hline $\mathbf{1}$ & 0.04 & 0.11 & 0.2 & 0.036 & 0.2 & 0.17 & 0.03 & 0.2 & 0.18 \\
& 0.02 & 0.78 & 1 & 0.023 & 0.45 & 0.11 & 0.037 & 0.43 & 0.11 \\
& 0.05 & 0.07 & 0.3 & 0.055 & 0.17 & 0.2 & 0.069 & 0.15 & 0.21 \\
\hline \multirow{2}{*}{$\mathbf{2}$} & 0.1 & 0.07 & 0.3 & 0.096 & 0.06 & 0.3 & 0.12 & 0.06 & 0.32 \\
\cline { 2 - 6 } & 0.1 & 0.07 & 1 & 0.096 & 0.06 & 0.95 & 0.12 & 0.06 & 0.8 \\
\hline $\mathbf{3}$ & 0.02 & 0.83 & 1 & 0.022 & 0.78 & 1 & 0.09 & 0.65 & 1 \\
\hline $\mathbf{4}$ & 0.05 & 0.15 & 1 & 0.046 & 0.18 & 1 & 0.095 & 0.13 & 1 \\
\hline
\end{tabular}

Figures (2) and (3) show the synthetic data and the calculated data using LM and SVD, respectively. The results show that the calculated data using the LM has a better fitting than the calculated data from the SVD method, but the calculated data from LM is more sensitive to the noise than those from the SVD method.

Figures (4) and (5) show the resulted model parameters from both inversion schemes. The model parameters were more resolved in the case of the SVD method than LM. Besides that, the LM inversionbased driven parameters are appeared to be more sensitive to the noise of the data. In the case of the linear inversion problem, the thickness of layers can be identified by notice the change in petrophysical parameters ranges within each layer, therefore, the resolution of SVD results can be more helpful than those of the LM method to detect the thickness of different layers. The GWC has been detected at depth of $9 \mathrm{~m}$ by both inversion methods. Both methods detect that the model has 4 main layers, layer 1 is shaly-sand $(0-5 \mathrm{~m})$, layer 2 is sand $(5-15 \mathrm{~m})$, layer 3 is shale $(15-20 \mathrm{~m})$, and layer 4 is sand $(20-25 \mathrm{~m})$. 
Also, both methods are sensitive to fluid change and detect that layer 2 consists of 2 parts the upper part is occupied by hydrocarbon, and the lower part is occupied by water. Figures (6a and 6b) show the convergence of both inversion schemes. The SVD starts to converge as the LM but needs more iterations until all the calculated well-logging data converge, which could be reflected from the idea of test the sensitivity of parameters changes with a certain well-log tool. The number of iterations needed for the convergence in the case of the LM method is around 10 iterations, while the convergence needed in the case of the SVD method is 10 to 50 to obtain convergence in all calculated well log data.

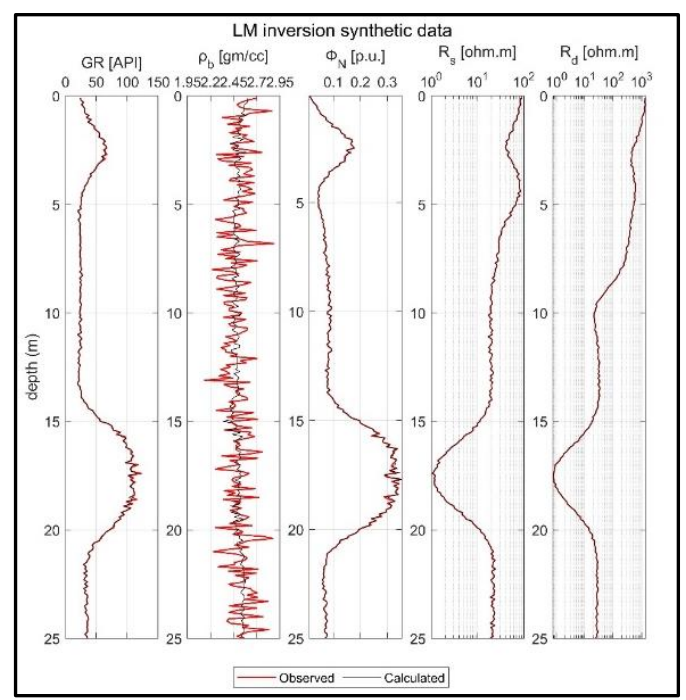

Figure 2. Observed synthetic data compared to the calculated data using LM inversion scheme (iteration 50).

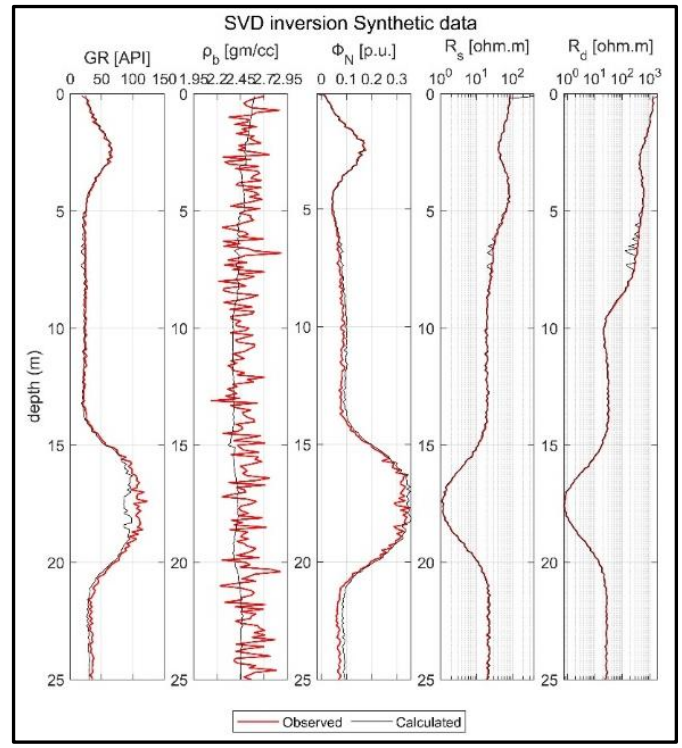

Figure 3. Observed synthetic data compared to the calculated data using SVD inversion scheme (iteration 50). 


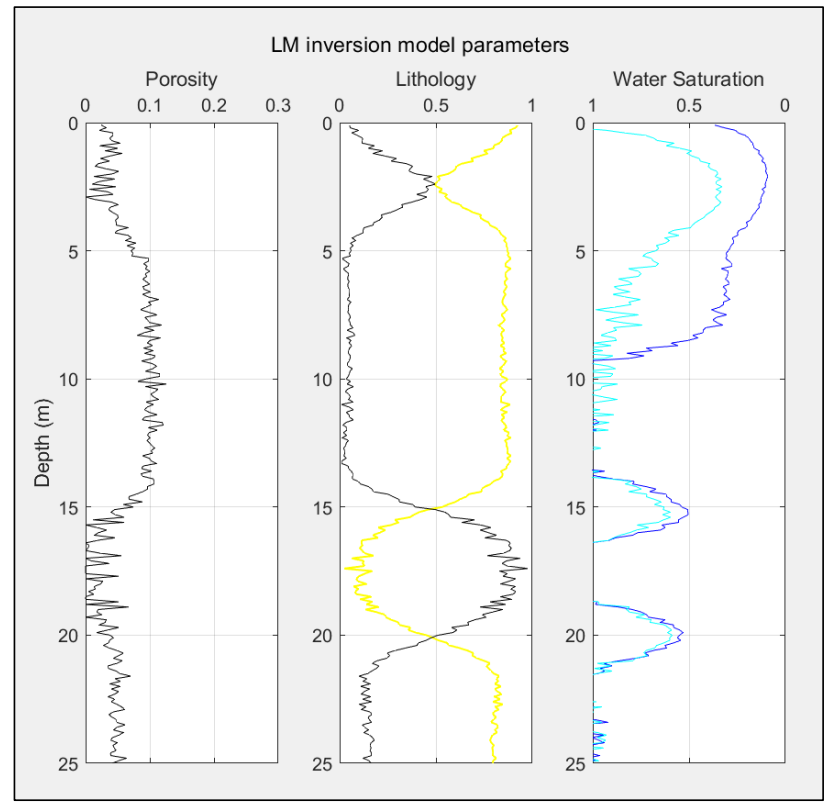

Figure 4. Model parameters derived from LM-based inversion (in lithology track, black is the shale fraction while the yellow is the sand fraction, while in water saturation track, blue is water saturation fraction in the uninvaded zone and cyan in the invaded zone).

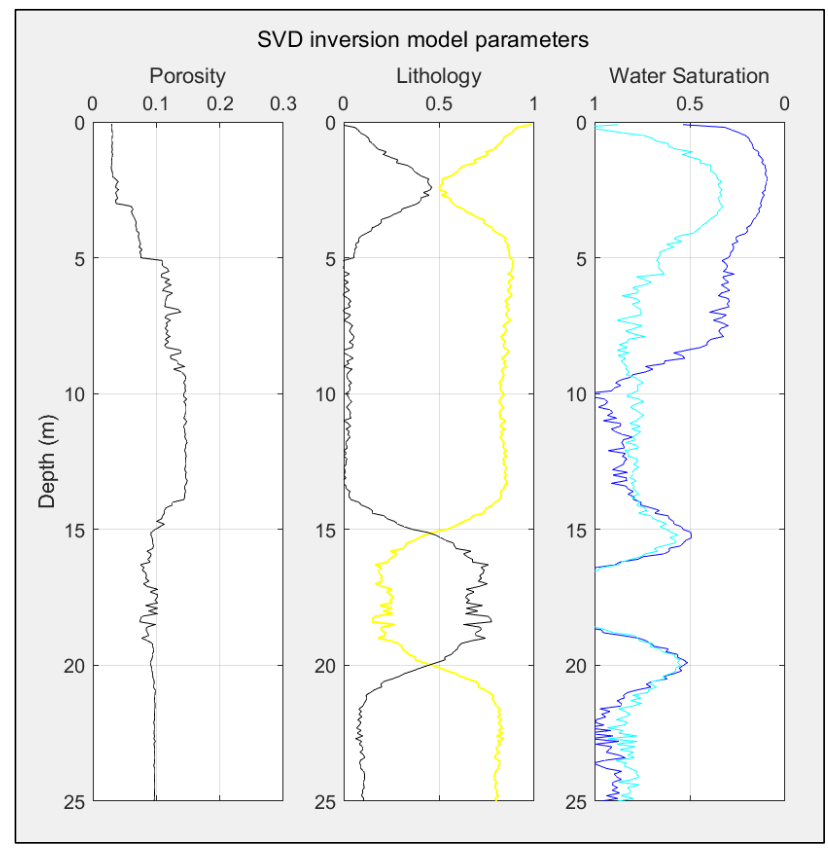

Figure 5. Model parameters derived from SVD-based inversion (in lithology track, black is the shale fraction while the yellow is the sand fraction, while in water saturation track, blue is water saturation fraction in the uninvaded zone and cyan in the invaded zone). 


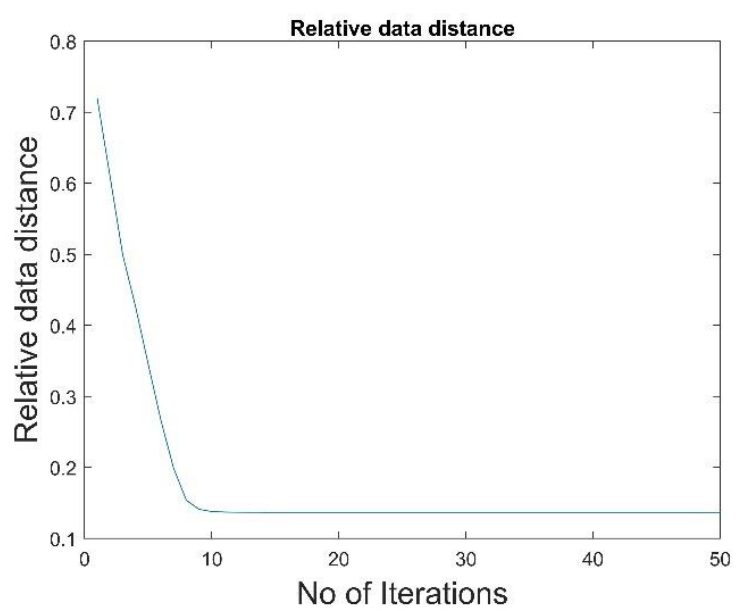

(a)

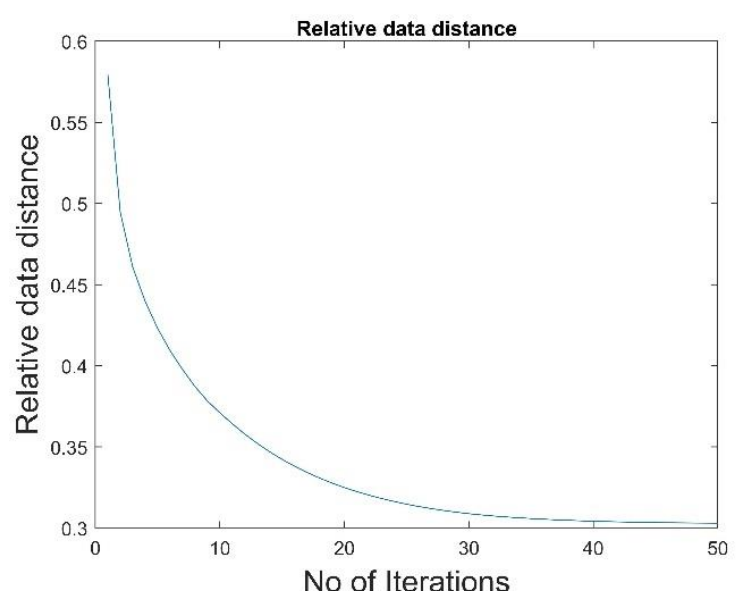

(b)

Figure 6. Relative data distance (a) LM-based Inversion and (b) SVD-based Inversion.

\subsection{Field data}

After LM and SVD schemes have been examined using synthetic data, this research implemented those methods to field data. The field data is well-logging data from a gas field located in the northwestern part of Egypt. From the regional geology of the area, the reservoir rocks consist mainly of tight sandstone with siltstone streaks which affect the quality of the reservoir and increase the heterogeneity inside it. The drilling information provided that the thickness of the reservoir is almost $170 \mathrm{~m}$ with hydrocarbons (gas) and water were occupied in its pore spaces. the data vector of this well is $\mathrm{d}=\left[G R, \emptyset_{N}, \rho_{b}, R_{s}, R_{d}\right]$.

As pre-mention, the results of the linearized inversion problem depend on the initial model, if the initial model is too far from the answer, it may be trapped in a local minimum. Therefore, the initial parameters have been chosen concerning the quick look interpretation of the well-logging data. The permeable intervals (low GR) have been given porosity ranges from 0.1 to 0.15 and water saturation of 0.5 , while the impermeable intervals (high GR) have been given porosity ranges from 0.06 to 0.08 and water saturation of 0.8 . In the preconditioning step, the measured data have been filtered using a median filtered of degree 10.

Figures (7) and (8) show the field data and calculated data using LM and SVD inversion methods, respectively. Both methods show an excellent fitting between field and calculated well-logging data with relatively high fitting in the case of LM-based inversion. Figures (9) and (10) show the model parameters resulted from both LM and SVD inversion schemes. The results have a significant notification; the SVD-based inversion can derive the same petrophysical parameters of LM-based inversion without using the trial-and-error method to pick the valid damping factor. Both methods are sensitive to the fluid presence, whereas, the GWC has been detected by both methods at depth of $65 \mathrm{~m}$.

Figures (11a) and (11b) show the relative data distance of both inversion schemes. The SVD-based inversion starts to converge from iteration 10 to 40, while the LM-based inversion starts to converge from iteration 14 to 20 . The SVD code shows more stability than the LM one. The convergence behavior of the LM shows a sharp decrease of the relative data distance reached 0.03, while in the case of SVD the convergence behavior shows a logarithmic decrease of the relative data distance which shows 
stability at a relative data distance of 0.21 . Both methods have a good fitting, but the SVD failed at some points at depth beyond 160 to deliver a good fitting which is responsible for a higher data distance.

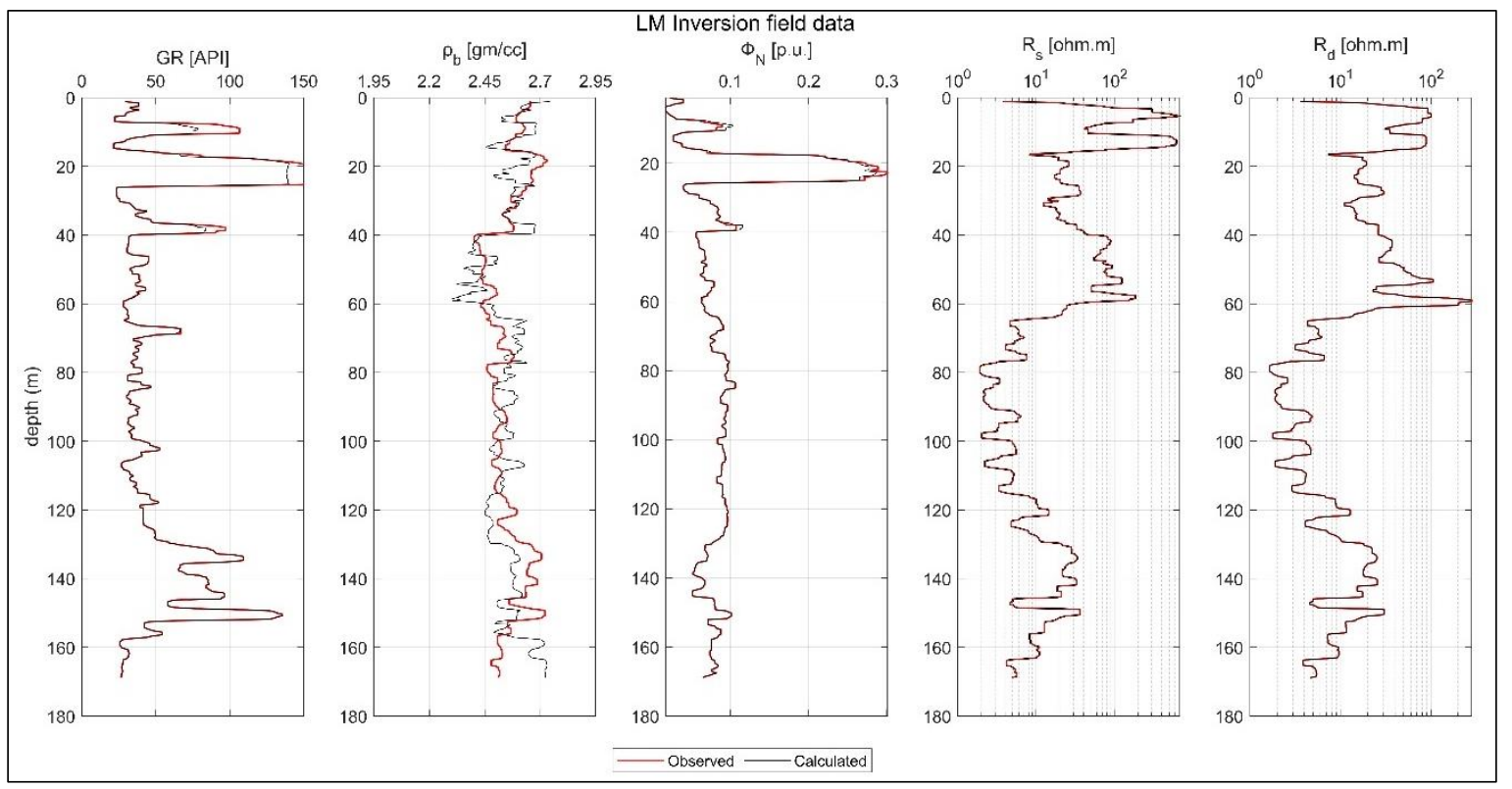

Figure 7. Observed Field data compared to the calculated data using LM inversion scheme (iteration 50).

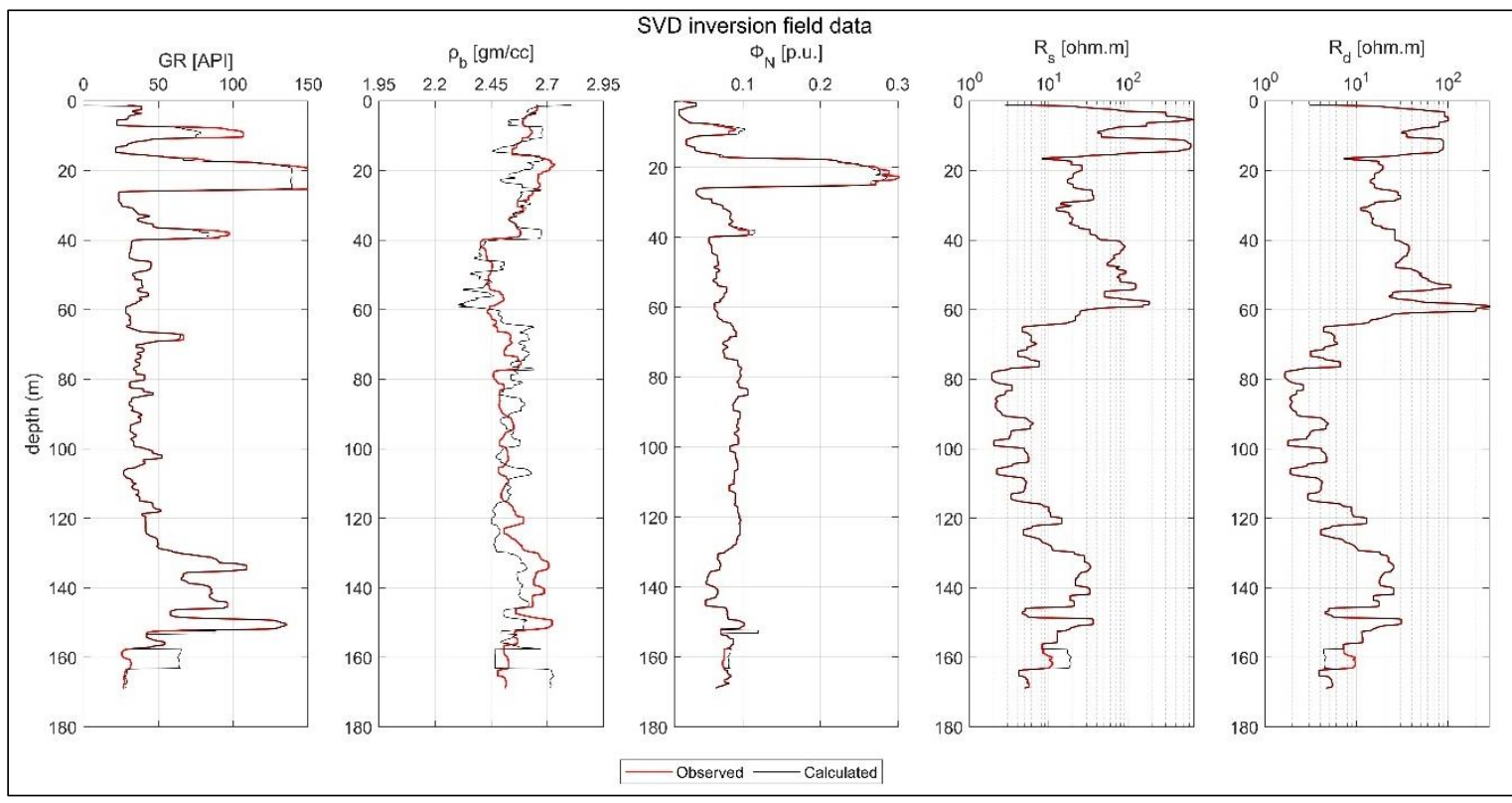

Figure 8. Observed Field data compared to the calculated data using SVD inversion scheme (iteration50). 


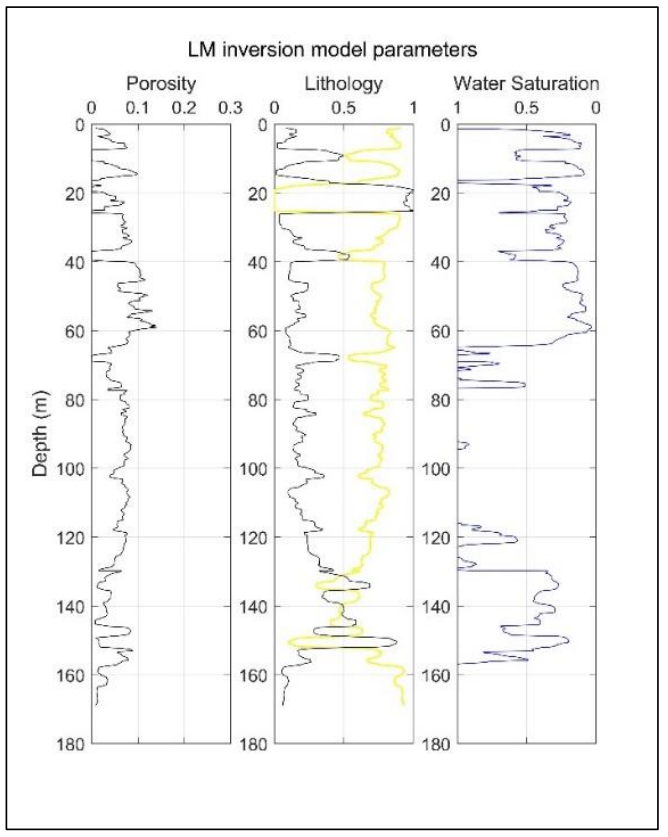

Figure 9. Model parameters derived from LM-based inversion (in lithology track, black is the shale fraction while the yellow is the sand fraction, while in water saturation track, blue is water saturation fraction in the uninvaded zone and cyan in the invaded zone).

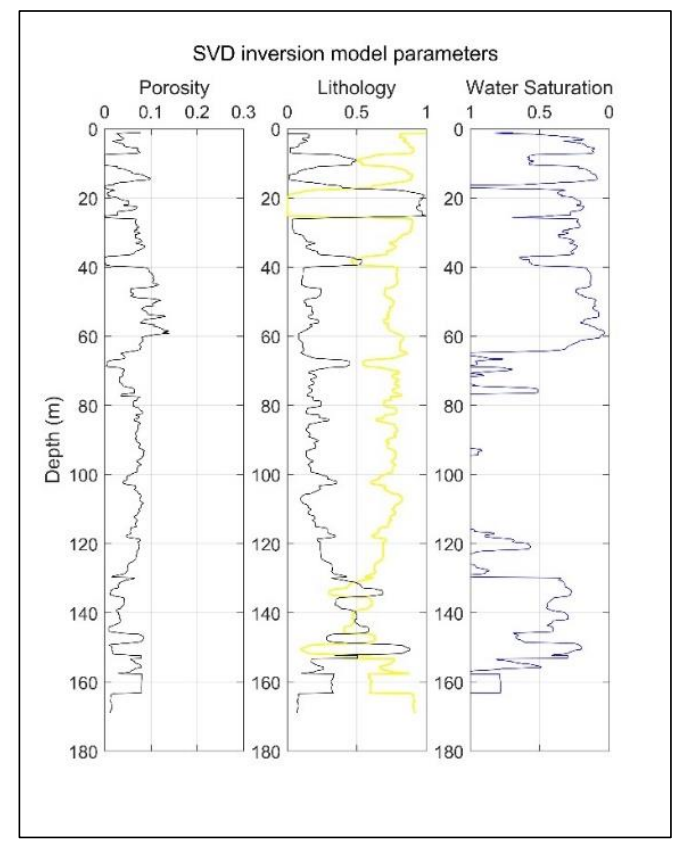

Figure 10. Model parameters derived from SVD-based inversion (in lithology track, black is the shale fraction while yellow is the sand fraction, while in water saturation track, blue is water saturation fraction in the uninvaded zone and cyan in the invaded zone). 


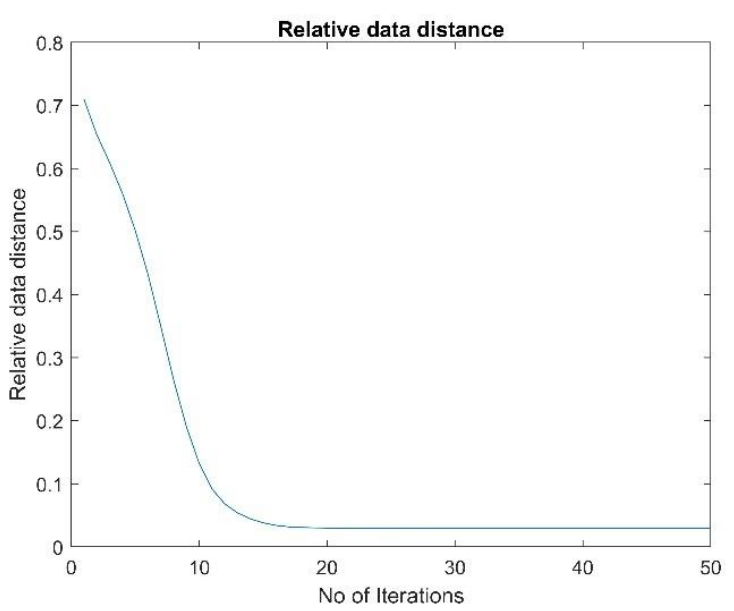

(a)

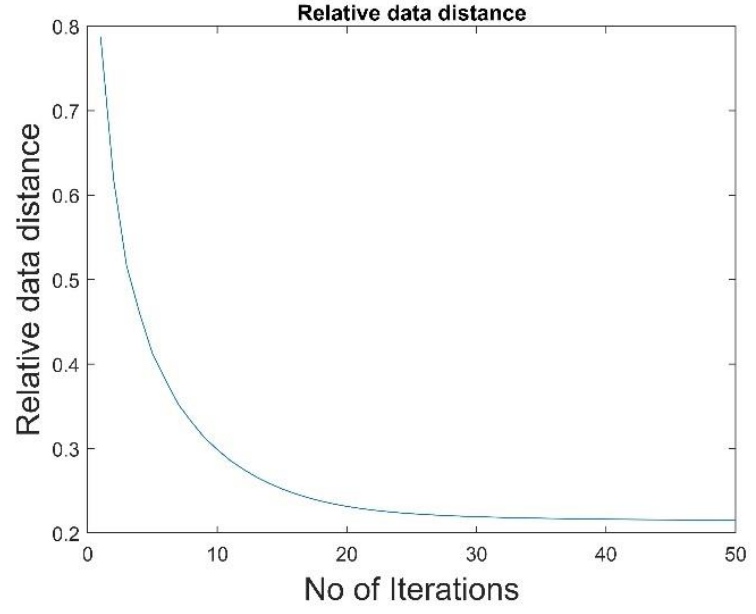

(b)

Figure 11. Relative data distance (a) LM-based Inversion and (b) SVD-based Inversion.

Figure 12 shows the comparison between the derived porosity from the SVD and LM methods and the projected cored porosity. The comparison shows that the porosity derived from SVD coincides with the LM porosity with some misfit points at the deeper parts. The predicted porosity is reliable at some depth intervals, which have been validated using the core porosity values.

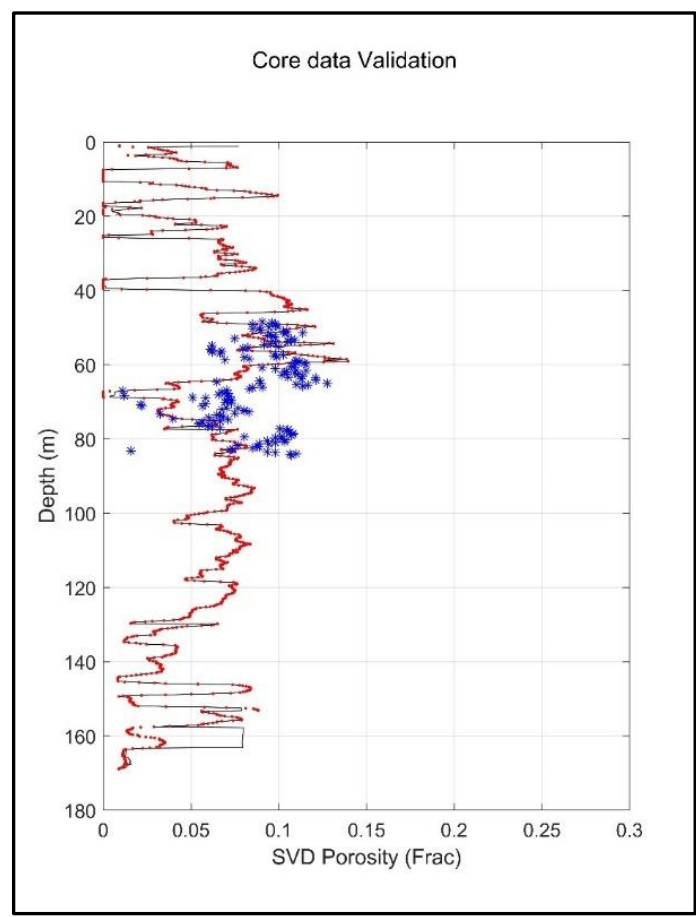

Figure 12. Projection of core porosity (blue stars) on the porosity derived from both SVD (Black line) and $L M$ (red dots) schemes. 


\section{Discussion}

Considering the synthetic data contaminated by $5 \%$ Gaussian noise, the fitting between synthetic and calculated well-logging data is good. The LM-based inversion shows relatively better data fitting than SVD-based. The degree of fitting and the iterations number needed for the convergence depending on the damping factor used in both methods, the LM-based method used the trial-and-error techniques to estimate the amount of damping factor which has been chosen to be high and multiplied by a factor less than one to damp the calculation within each iteration. The results from the SVD-Based method are less sensitive to the amount of noise than those of the LM-based. The SVD-based inversion method tried to solve the overdetermined well-logging problem using the concept of RIDGE to overcome forcing the model parameters to enhance the fitting in the same step, where the damping factor has been chosen in each step as a function of the eigenvalues of the Jacobian matrix, then tested with next calculated data before accepting the change in those parameters. This inner loop provides stability in the inversion MATLAB code as well as avoids the underestimated parameters. In the case of the LM-based inversion, the development of convergence was smooth and steady sharply after 10 iterations, while in the SVDbased inversion scheme the development of convergence was smooth and steady at iteration no. 40 .

In the field data, in SVD, eigenvalue selection is considered a vital way because the method considered more the matrix's sensitiveness. The results from both schemes show that the SVD-based inversion scheme can be used to derive the petrophysical parameters by using the damping factor derived from the sensitivity matrix where in the case of the LM-based method the results depend on the value of damping factor which was chosen based on the trial-and-error method. Furthermore, the SVD-based inversion method could overcome the heterogeneity change within the reservoir which affects the response of the well-logging tool. The field data is considered as a deep reservoir that is affected by the overburden weight, therefore, the response of some logs such as density $\log$ can be deceptive and produce fake inversion results. As the thickness of the layers in the local inversion techniques can be noticed from the change in the petrophysical parameters from one layer to another, the SVD-based method can be recommended in the heterogeneous reservoir because of the consideration of the details of the Jacobian matrix's sensitiveness.

\section{Conclusion}

This research study examined LM-based and SVD based inversion methods, as well as compared them using synthetic data contaminated by 5\% Gaussian noise and a deep heterogeneous reservoir. Both methods are good to be used in the synthetic data, where the LM converges faster than SVD. In the case of the field data, a method of choosing and testing the damping factor is recommended in the case of using the LM-based inversion to control the underestimation or the overestimation in petrophysical parameters. In the case of the SVD-based inversion method, the selection of the eigenvalues enhanced the resulted petrophysical parameters by considering the Jacobian matrix's sensitiveness. Both methods predicted the presence of hydrocarbon above the depth of $65 \mathrm{~m}$. The projection of the porosity core points and the derived porosity data using the SVD method showed a good fitting.

\section{Acknowledgment}

The author would like to express his deep gratitude to the Egyptian cultural affairs and missions' sector for their continuous financial and moral support. The researcher is funded by a scholarship under the joint between Egypt and Hungary. 


\section{References}

[1] Dobróka, M., Szabó, N. P., Tóth, J. and Vass, P. (2016). Interval inversion approach for an improved interpretation of well logs. Geophysics, 81(2), D155-D167. https://doi.org/10.1190/GEO2015-0422.1

[2] Szabó, N. P., Dobróka, M. (2020). Interval inversion as innovative well log interpretation tool for evaluating organic-rich shale formations. J. Pet. Sci. Eng., 186(November), 106696. https://doi.org/10.1016/j.petrol.2019.106696

[3] Szabó, N. P., Dobróka, M. (2013). Extending the application of a shale volume estimation formula derived from factor analysis of wireline logging data. Math. Geosci., 45(7), 837-850. https://doi.org/10.1007/s11004-013-9449-2

[4] Dobróka, M., Szabó, N. P. (2011). Interval inversion of well-logging data for objective determination of textural parameters. Acta Geophys., 59(5), 907-934. https://doi.org/10.2478/s11600-011-0027-z

[5] Szabó, N. P. (2018). A genetic meta-algorithm-assisted inversion approach: hydrogeological study for the determination of volumetric rock properties and matrix and fluid parameters in unsaturated formations. Hydrogeol. J., 26(6), 1935-1946. https://doi.org/10.1007/s10040-0181749-7

[6] Drahos, D. (2005). Inversion of engineering geophysical penetration sounding logs measured along a profile. Acta Geod. Geophys. Hungarica, 40(2), SPEC. ISS., 193-202. https://doi.org/10.1556/AGeod.40.2005.2.6

[7] Szabó, N. P. (2011). Shale volume estimation based on the factor analysis of well-logging data. Acta Geophys., 59(5), 935-953. https://doi.org/10.2478/s11600-011-0034-0

[8] Alberty, M., Hashmy, K. H.: Application of ULTRA to log analysis, 1984 SPWLA 25th Annual Logging Symposium, pp. 1-17.

[9] Ben-yaacov, I., Roig, F. (2006). Index notation for vector calculus. pp. 1-10.

[10] Menke, W. (1984). Geophysical data analysis: Discrete inverse theory. Academic Press Inc.

[11] Szabó, N. P. (2012). Dry density derived by factor analysis of engineering geophysical sounding measurements. Acta Geod. Geophys. Hungarica, 47(2), 161-171. https://doi.org/10.1556/AGeod.47.2012.2.5

[12] Marquardt, D. W. (1963). An algorithm for least-squares estimation of nonlinear parameters. Journal of the Society for Industrial and Applied Mathematics, Q. Appl. Math., 11(2), 431-441. https://doi.org/10.1137/0111030

[13] Heriyanto, M., Srigutomo, W. (2017). 1-D DC resistivity inversion using singular value decomposition and Levenberg-Marquardt's inversion schemes. Journal of Physics: Conference Series, 877(1). https://doi.org/10.1088/1742-6596/877/1/012066

[14] Meju, M. A. (1992). An effective ridge regression procedure for resistivity data inversion. Comput. Geosci., 18(2-3), 99-118. https://doi.org/10.1016/0098-3004(92)90079-7

[15] Ekinci, Y. L., Demirci, A. (2008). A damped least-squares inversion program for the interpretation of Schlumberger sounding curves. J. Appl. Sci., 8(22), 4070-4078. https://doi.org/10.3923/jas.2008.4070.4078

[16] Arneson, K., Hersir, G. P. (1988). One dimensional inversion of Schlumberger resistivity soundings: Computer Program, Description and User's Guide. 